be discovered which will show that the theory of natural selection is not fully adequate to account for evolution".

The essence of Whyte's book is the conclusion that the conditions of biological organization restrict, to a finite discrete spectrum, the possible avenues of evolutionary change from a given starting point. The nature of life limits its variation and is one factor directing phylogeny. Thus the mutations of which the consequences reach the Darwinian test have already been sifted by an internal selection process. This internal selection restricts the directions of evolutionary change by internal organizational factors. The author says "there may be no mutations which ean be fully aseribed to chance", and he quotes Bachelard (1953) that there is implicit in quantum mechanics a structuring tendency for complex systems to form more complex forms of ordering. He then suggests, though obviously with some doubts, that this could perhaps be the basis of internal selection. Thus, the attempt is made to complement the Darwinian principle of external adaptive selection by a new type of selection acting directly on changes inside the organism at the molecular and chromosomal levels. Under this principle, random mutations are subjected to a selection process dependent on their compatibility with the internal organization and processes of the organism, before the adult faces environmental competition. It is his view that biological order, co-ordination and organization have equal importance with random statistical competition and that this concept is, in a radical philosophical sense, complementary to Darwin's principle and has, therefore, significant implications for the psychological and social sciences as well as for biological sciences. Medawar (1960) is quoted as having suggested that there is in systems of polymers a 'repetitiousness' or tendency towards elaboration, so that ever more complex sets of genetical instructions are offered for trial and that this may provide a basis for advancing complexity; and I think the general response for molecular biologists to Whyte's views will be favourable.

The book is brief, trenchant and profound. The fact that it may not contain any ideas which are absolutely new is no weakness; rather it is in this that much of its strength lies. Whyte is to be congratulated on having brought togethor and unified in discussion a number of ideas that have been somewhat on the fringe of biological thought in recont years. All who are interested in the fundamental ideas of unity, ordering, complexity, adaptiveness and directiveness - and that is to say almost all biologists - should read this book. Once they have read it they are unlikely, whatever their response, to forget it. They may disagree on how good the evidence is for some of the conclusions and on how far ideas put forward have been adequately covered by earlier writers. They can hardly argue that the problems raised are other than topical and fundamental.

W. H. THORPE

\section{ADSORPTION FROM SOLUTION}

\section{Adsorption from Solutions of Non-Electrolytes}

By J. J. Kipling. Pp. xii +328. (London: Academic Press, Inc. (London), Ltd.; New York: Academic Press, Inc., 1965.) $73 s$.

TTHE understanding of adsorption from solution requires not only a knowledge of the dispositions of, and interactions between, the solution molecules in a surface phase, but also a knowledge of the corresponding situation in the bulk of the solution. Solution and liquid state theory are not, however, in a well-developed state, at least as compared to that for gases, and if only for this reason we may expect adsorption from solution to be a difficult field of investigation. The understanding of adsorption on to solid surfaces from solution requires in addition an intimate knowledge of the solid surface and its interactions with the liquid phase components. The complexities of such systems are obviously immense and it is scarcely surprising that adsorption from solution on to solids has the appearance of being a rather neglected subject. Adsorption from Solutions of Non-Electrolytes by J. J. Kipling, is the first book of its kind and reflects accurately the present state of the subject.

This book is almost entirely concerned with adsorption on to solids. There are sections on liquid-vapour and liquid-liquid interfaces, but these are included more for purposes of comparison than for their intrinsic interest. Apart from this limitation, the book is very comprehensive and is based on the extensive experience and work of the author in this field. The early chapters describe the methods of investigating adsorption while the later ones are devoted to specific systems, and the results that are now available to us. The latter are presented systematically. Five chapters deal successively with adsorption from completely miscible liquid mixtures, from partially miscible liquids, of gases, solids and polymers from solution and of adsorption from multicomponent systems. Among the remaining chapters are descriptions of the kinetics of adsorption and the thermodynamics of adsorption. The book contains a considerable amount of data and probably all the important references. The material is well presented and very readable.

Although it will be primarily of interest to research workers in surface chemistry, there are many sections which can readily be used for undergraduate instruction. In my opinion, this is an excellent and much-needed book and is to be wholeheartedly recommended. Tho only disappointing feature is the price.

\section{A. HAYDON}

\section{ELECTRONS AND HOLES AT THE SURFACE}

\section{Semiconductor Surfaces}

By A. Many, Y. Goldstein and N. B. Grover. Pp. xvi+ 496. (Amsterdam: North-Holland Publishing Company. 1965.) $110 s$.

$7 \mathrm{HE}$ advent of the transistor after the Second World War did more than revolutionize the electronics industry. It set solid-state physies on its feet. It provided, in the study of germanium and silicon, the first really critical test of the electronic theory of solids, one of the triumphs of quantum theory. It stimulated research into many aspects of the solid state. And it presented, in the $p-n$ junction, the first really simple example of an electrified double layer.

The great success of $p-n$ junction theory has rested largely on two simplifying features: the very low concentrations of the charged particles, and the fact that the electrons and holes move in the same medium on both sides of the junction. In much of the recent research on silicon and germanium surfaces these two advantages have been retained. Electrons and holes in the semiconductor remain, as a rule, the only mobile charge carriers. The bending of the bands at the surface, even in extreme cases of inversion or accumulation, is rarely so great as to introduce degeneracy. The amount of bending, expressed by the surface potential or the electric field normal to the surface, can be controlled at will by using an external electrode (the field effect) or by chemical means. Passage of current parallel to the surface is used to determine excess densities of electrons and holes, and to measure surface mobilities, which are lower than in the bulk. These and other techniques have been elaborated and applied with considerable success to silicon and germanium surfaces, and it is fair to say that we have by now reliable and detailed models both for clean surfaces and for real (oxidized) surfaces, at least up to and including the fast states on the inside of the oxide. The slow states on the outside present a more difficult problem. 